\title{
Steady supercritical Taylor vortex flow
}

\author{
By J. E. BURKHALTER† AND E. L. KOSCHMIEDER \\ College of Engineering, The University of Texas, Austin
}

(Received 5 September 1972)

Experiments studying steady supercritical Taylor vortex flow have been made using pairs of long cylinders with two different radius ratios, three fluids of different viscosities and three different end boundaries for the fluid column. The emphasis in these experiments is on the determination of the wavelength of the Taylor vortices and the size of the end rings. The wavelength which one measures in a finite cylinder differs from the wavelengths found theoretically for infinitely long cylinders. Provided that the end effeets were properly taken into account, the wavelength of singly periodic Taylor vortices in an infinitely long cylinder was found to remain constant between $T / T_{c}=1$ and $T / T_{c} \approx 80$ in experiments with radius ratios $\eta=0.505$ and $\eta=0.727$. Further studies of Taylor vortex flow at very high Taylor numbers, where the vortices are either doubly periodic or truly turbulent, showed that the wavelength increases under these conditions. However, the observed wavelengths were no longer unique but distributed statistically around a wavelength larger than the critical wavelength.

\section{Introduction}

Supercritical Taylor vortex flow is one of the principal objects of study in the current efforts to develop a nonlinear stability theory. The work on this topic has recently been reviewed by Stuart (1971). It is important that further experimental data be obtained so that the success of the theoretical endeavours can be verified. The information which follows most directly from an experiment with supercritical Taylor vortices concerns the size or the so-called wavelength of the vortices. The present investigation studies the wavelength of Taylor vortices which are established at the critical Taylor number $T_{c}$ and whose Taylor number is later increased quasi-steadily. The question of how the wavelength of a Taylor vortex may vary if the Taylor number $T$ is increased slowly has apparently not been investigated theoretically. However, information is available concerming the possible range of wavelengths for given supercritical $T$. The most recent theoretical investigation of the range of stable wavelengths, that is wavelengths for which the calculated Taylor vortex flow is stable to axisymmetric disturbances, has been made by Kogelman \& DiPrima (1970). Knowledge of the actual behaviour of the wavelength under supercritical conditions is uncertain; there are several contradictory statements concerning the wavelength in the experimental literature. Pai (1943), working with a short apparatus with radius

$\dagger$ Present address: Auburn University, Auburn, Alabama. 
ratio $\eta=R_{i} / R_{o}=0 \cdot 88$, observed a change from 6 to 4 rings at fairly high Taylor number. A reduction of the number of rings is equivalent to an increase in the wavelength. Schultz-Grunow \& Hein (1956) have published photographs which likewise show a reduction of the number of vortices when the Taylor number was increased to very high values. They used a long apparatus with $\eta=0 \cdot 84$. An increase of the wavelength in the case of doubly periodic flow in a long apparatus with $\eta=0.874$ has, furthermore, been observed by Coles (1965). The first deliberate measurements of the wavelength $\lambda$ seems to have been made by Donnelly \& Schwarz (1965). Their measurements indicate a small decrease of $\lambda$ up to $T=2100$ in a set-up with $\eta=0.95$. However, when $\eta$ was either 0.90 or 0.85 a definite increase of $\lambda$ was observed. When $\eta=0.85$ the increase of $\lambda$ was observed up to $T=45000$, corresponding to $T / T_{c}=25$. In this case the flow had a small 'harmonic distortion'. On the other hand Snyder (1969), working with singly periodic flow in a long apparatus with $\eta=0.5$, found that "the variation of wavelength with Taylor number is linear and the slope is exceedingly small and negative".

It is usually assumed that the similarity between Bénard convection and Taylor vortex flow holds for supercritical conditions too. From this point of view one should expect an increase of the wavelength of Taylor vortices with Taylor number, since it is an unambiguous result of experimental work that the wavelength of Bénard convection increases with increased Rayleigh number. For a review of the relevant investigations see Koschmieder (1973), who cites ten papers all of which demonstrate the increase of $\lambda$. An actual increase of the wavelength of Taylor vortices would agree with the fairly clear-cut evidence obtained by Schultz-Grunow \& Hein (1956). However, it remains to be shown in much more definite form under which conditions and in which interval of Taylor numbers the wavelength changes and by how much.

\section{Description of the apparatus}

A schematic section through the apparatus is shown in figure 1. The working fluid was placed in the space between two concentric cylinders mounted vertically. The outer cylinder was held stationary at all times while the inner cylinder was rotated around the axis of the apparatus. The outer cylinder was a pyrex glass pipe which was borrowed from the Hydrodynamics Laboratory of the University of Chicago, and was the same as that used by Donnelly \& Fultz (1960). The inside surface was ground smooth so that the internal radius was 6.285 $\pm 0.006 \mathrm{~cm}$. The length of the glass cylinder was $91.44 \mathrm{~cm}$ and the wall thickness was about $7 \mathrm{~mm}$.

Two inner cylinders were used in the experiments. The first cylinder was brass and was machined to a diameter of $6 \cdot 350 \pm 0 \cdot 003 \mathrm{~cm}$. At any particular location no variation in the diameter was detectable with a dial indicator accurate to $0.001 \mathrm{in}$. The variation in diameter of $\pm 0.003 \mathrm{~cm}$ occurred because of machining taper over the length of the cylinder. With this cylinder a gap width of $3 \cdot 110$ $\pm 0.006 \mathrm{~cm}$ was obtained; the radius ratio $\eta=R_{i} / R_{o}$ was then 0.505 . The second inner cylinder was bronze and was machined to a diameter of $9 \cdot 144 \pm 0.003 \mathrm{~cm}$, 


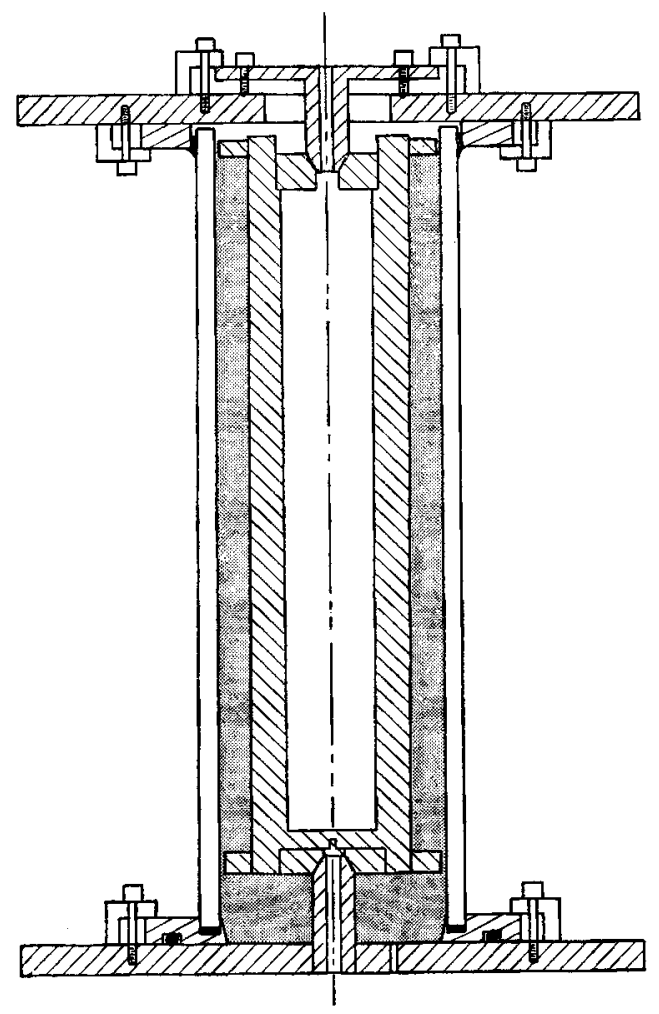

Figure 1. Section through the apparatus. III, inner cylinder assembly; /II/, support frame assembly; UIIII, support flanges for outer glass cylinder;

where again the variation in diameter was due to machining taper. The gap width was in this case $1.713 \pm 0.006 \mathrm{~cm}$ and $\eta=0.727$. Both cylinders were polished to a near mirror finish. The fluid column length was $87.30 \mathrm{~cm}$, with both cylinders. There was space for 14 rings (28 cells) if $\eta=0.505$ and 25 rings if $\eta=0 \cdot 727$.

The inner cylinder was turned by a Graham drive. Using various gear belt pulley systems, the angular velocity $\Omega$ of the inner cylinder was continuously variable up to $600 \mathrm{rev} / \mathrm{min}$. This system could maintain any given rotation rate to an accuracy of $0.4 \%$ of the mean angular velocity. Precise measurement of the time for one revolution, i.e. the angular velocity of the inner cylinder, was obtained electronically with a microsecond counter. The angular velocity of the inner cylinder could be changed without sudden finite steps by using a small motor to turn the speed control of the Graham drive. The Taylor number $T$ for an apparatus with radius ratio $\eta$ is given by the formula (Roberts 1965)

$$
T=\frac{2 \eta^{2}}{1-\eta^{2}} \frac{\Omega^{2} d^{4}}{\nu^{2}}
$$

with $d$ the gap width and $\nu$ the kinematic viscosity.

Three different fluids were used in the experiments, two being commercial 


$\begin{array}{ccccc} & \begin{array}{c}\text { Temperature } \\ \left({ }^{\circ} \mathrm{C}\right)\end{array} & \begin{array}{c}\text { Fluid 1 } \\ \text { Glycerol }\end{array} & \begin{array}{c}\text { Fluid 2 } \\ \text { Medium oil }\end{array} & \begin{array}{c}\text { Fluid 3 } \\ \text { Heavy oil }\end{array} \\ \text { Kinematic viscosity } & \mathbf{2 2} & 0.029 & 0 \cdot 448 & 1.460 \\ \left(\mathrm{~cm}^{2} \mathrm{~s}^{-1}\right) & \mathbf{2 3} & \mathbf{0 . 0 2 6} & \mathbf{0 . 4 2 4} & \mathbf{1 . 4 2 0} \\ & 24 & 0.024 & 0 \cdot 400 & \mathbf{1 . 3 8 0}\end{array}$

TabLe 1. Viscosity of fluids used

(Gulf Harmony) machine oils and one a water-glycerin mixture. The viseosity of these fluids was measured and is listed in table 1.

The viscous diffusion times $t=d^{2} / \nu$ for the case $\eta=0.727$ were $108 \mathrm{~s}, 6.9 \mathrm{~s}$ and $2 \cdot 0 \mathrm{~s}$ for fluids 1,2 and 3 respectively. In all experiments with the fairly viscous fluids 2 and 3 , the flow was always in equilibrium when the increase of the rotation rate was controlled by the secondary gear motor and the acceleration rate of the inner cylinder was either $0.0014 \mathrm{rad} / \mathrm{s}^{2}$ or $0.00077 \mathrm{rad} / \mathrm{s}^{2}$. Also, in all cases, when increases of $\Omega$ were made by hand and were consequently unsteady, the fluid adjusted to equilibrium in around a minute. Steady experiments, when referred to here, are concerned with such conditions. With fluid 1, equilibrium was not established as easily since the diffusion time is so much longer. Since, on the other hand, with fluid 1 the range of possible Taylor numbers is very much larger, it is practically impossible to go through the entire Taylor number range with the fluid in equilibrium all the time. Hence, $\Omega$ was increased stepwise, followed by long waiting periods in which the fluid obtained equilibrium. Finally, at very high Taylor numbers, when the flow was turbulent, the diffusion time of fluid 1 decreased drastically. Waiting times of about a minute then assured equilibrium.

The fluid motion was made visible by adding aluminium powder to the liquids. This tracer makes the fluid appear dark (black) if the motions in the fluid are in the radial direction towards or away from the observer. The fluid appears light (silvery) if the fluid is moving parallel to the observer.

A cathetometer accurate to $\pm 0 \cdot 1 \mathrm{~mm}$ was used to measure the size or height of the rings or cells. Usually five cells or rings were measured: the two end cells, the rings adjacent to the end cells (or rings) and a ring in the middle of the fluid column. There were experiments in which the sizes of all the rings in the column were measured. It was found that the five measurements mentioned above were representative and were adequate for experimental analyses.

A sample of measurements of the vertical extent $\delta$ of all cells is given in table 2 . Results for four rings adjacent to the upper and lower boundaries of the fluid are listed together with those for two rings in the middle of the column. The average length of all rings in the fluid column is listed with the standard deviation of the measurements of all rings. The first line in table 2 shows results for the large gap, where the accuracy of the measurement is best and the end effects are large. It shows that the second ring at both boundaries is about $1 \%$ larger than the average ring size. With the small gap the rings adjacent to the end ring usually do not differ from the average ring size within the error of measurement. In other words, the end boundaries affect the size of the second ring very little. All photographs of the vortices substantiate this. The variation of the size of the second 


\begin{tabular}{|c|c|c|c|c|c|c|c|c|}
\hline \multirow[b]{2}{*}{$\eta$} & \multirow[b]{2}{*}{$T / T_{c}$} & \multirow[b]{2}{*}{ Rings: } & \multicolumn{3}{|c|}{ Top } & & \multicolumn{2}{|c|}{ Middle } \\
\hline & & & 1 & 2 & 3 & 4 & 1 & 2 \\
\hline $\begin{array}{l}0.505 \\
0.727\end{array}$ & $\begin{array}{l}4 \cdot 23 \\
4 \cdot 23\end{array}$ & $\delta(\mathrm{cm})$ & $\begin{array}{l}5 \cdot 80 \\
3 \cdot 84\end{array}$ & $\begin{array}{l}5 \cdot 07 \\
3 \cdot 45\end{array}$ & $\begin{array}{l}5 \cdot 04 \\
3 \cdot 48\end{array}$ & $\begin{array}{l}5 \cdot 07 \\
3 \cdot 46\end{array}$ & $\begin{array}{l}5 \cdot 02 \\
3 \cdot 39\end{array}$ & \\
\hline
\end{tabular}

Bottom

$\begin{array}{cccccccc}4 & 3 & 2 & 1 & \bar{\delta} & \lambda_{\text {eff }} & \text { Fluid 2 } & \text { Rings } \\ 5 \cdot 00 & 5 \cdot 00 & 5.08 & \mathbf{5 . 9 4} & \mathbf{5 . 0 2} \pm \mathbf{0 . 0 3} & \mathbf{1 \cdot 6 1} & \text { Resting end } & 17 \\ 3 \cdot 44 & 3 \cdot 51 & 3.48 & 3 \cdot 82 & 3 \cdot 46 \pm 0.03 & 2 \cdot 02 & \text { boundaries } & 25 \\ & & & & \text { TABLE } 2 & & & \end{array}$

rings is at most about $1 \%$ and affects the measured average size of all rings by at most $0.15 \%$, which is small compared with the standard deviation of the measurements of all rings.

Further details of the apparatus concerning the very precise alignment of the cylinder axes, the temperature control and the mounting of the apparatus cannot be discussed here but can be found in Burkhalter (1972).

\section{Wavelength definition}

Since experiments can only be made in cylinders of finite length, an experimentally measured wavelength differs from the theoretical wavelength $\lambda$, which is defined as the ratio of the vertical extent of one ring to the gap width in a cylinder of infinite length. For experimental investigations, two definitions of the wavelength must be considered. The first definition of the wavelength for a finite fluid column makes no provisions for end effects, and is

$$
\lambda_{\mathrm{fi}} \equiv L / N d,
$$

where $L$ is the fluid column length, $N$ is the number of rings ( = half the number of cells) and $d$ is the gap width. At the onset of instability, $\lambda_{\mathfrak{f}}$ can differ from the theoretical wavelength $\lambda$ by the amount $\Delta \lambda / \lambda= \pm 100 / 2 N \%$, because an integral number of rings $N$ has to fill the fluid column, the so-called quantization condition. The wavelength defined by (2) cannot change unless the number of Taylor vortices changes. Also, the uncertainty $\Delta \lambda$ changes only with $N$, regardless of whether the conditions are critical or supercritical. The wavelength as defined by (2) approximates the wavelength in an infinite cylinder whenever $L / d$ is large. Variations in the end cell size then have increasingly less bearing on the other cells in the fluid column.

The second definition of an experimentally measured wavelength takes into account changes in the end cell size, as well as changes in the number of rings. The effective wavelength is defined by the formula

$$
\lambda_{\text {eff }} \equiv(L-E) / N^{\prime} d=\delta / d,
$$


where $E$ is the sum of the size of the two end cells or rings, $\delta$ the actually measured vertical length of a ring free from end effects and $N^{\prime}$ is the number of rings excluding the end rings. Great care must be exercised in interpreting results obtained with $\lambda_{\text {eff }}$ since the effective wavelength is a function of the end cell size $E$. The effective wavelength can vary continuously since the end cell size can vary continuously with Taylor number. However, changes in the end cell size are not necessarily caused by changes in the size of Taylor vortices but rather are due to the boundary conditions at the ends of the fluid column. Also, the uncertainty $\Delta \lambda$ due to quantization is only $\pm \lambda / 2 N$ at the onset of instability. For supercritical conditions, $\Delta \lambda$ is a function of $\Delta E$ too. However, as does $\lambda_{\mathrm{fi}}$, the effective wavelength $\lambda_{\text {eff }}$ approximates the theoretical $\lambda$ if $L / d$ is sufficiently large.

\section{Investigation of the end cells}

Since the effective wavelength is dependent upon the size of the end cells a systematic study of the variation of the size of the end cells with Taylor number is necessary. In the present experiments three different end boundaries were considered: (i) rotating solid end boundaries, (ii) non-rotating solid end boundaries, and (iii) a free-surface boundary on top of the fluid column in conjunction with either a rotating or non-rotating boundary at the bottom.

A rotating solid end boundary means that the end plate, which covers the entire fluid gap except for a small space less than $1 \mathrm{~mm}$ wide at the outer cylinder, rotates with the same angular velocity $\Omega$ as the inner cylinder. A rotating boundary always produced a source (fluid moving radially outwards) at the end boundary. Consequently a single cell or half a ring formed at the ends of the fluid column. A typical example of such an end cell and its growth with increased $\Omega$ can be seen at the bottom of the fluid column in figures $2(a)-(e)$ (plate 1). The size $\lambda_{E}$ of the end cell increased with Taylor number as long as the flow was singly periodic. In experiments where both the top and bottom end boundaries were rotating, both end cells increased in size uniformly. In all experiments with symmetric end boundaries the fluid column was symmetric about the horizontal centre-line. Measured values of the size $\lambda_{E}$ of the rotating end cell, normalized with the gap width, are shown in figures $3(a)$ and $(b)$ for two different $\eta$ and the different fluids. It is obvious that $\lambda_{E}$ is proportional to $\left(T / T_{c}\right)^{\frac{1}{2}}$. The only deviation from the linear relationship occurred with the wide gap and fluid 2 when the upper surface was free. Within experimental error it makes no difference to the growth of the end cells whether both end plates rotate or only the lower end plate rotates and the upper surface is free. However, it is quite apparent that the radius ratio $\eta$ has a profound effect on the cell size. With a larger gap ( $\eta$ smaller) the end cells grow more rapidly. This might imply that, as $\eta$ approaches $1 \cdot 0$, the end cell ceases to grow. The equations governing the cell growth rate in figure 3 for $\eta=0.505$ are given by

$$
d \lambda_{E} / d \xi=A
$$

where $\xi=\left(T / T_{c}\right)^{\frac{1}{2}}$ and $0.39 \leqslant A \leqslant 0 \cdot 46$. For $\eta=0.727$, it follows that

$$
d \lambda_{E} / d \xi=B
$$




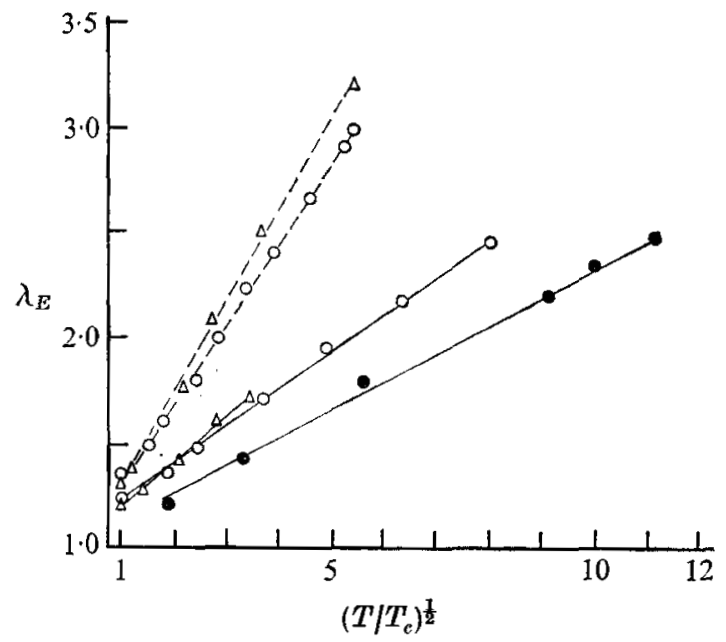

(a)

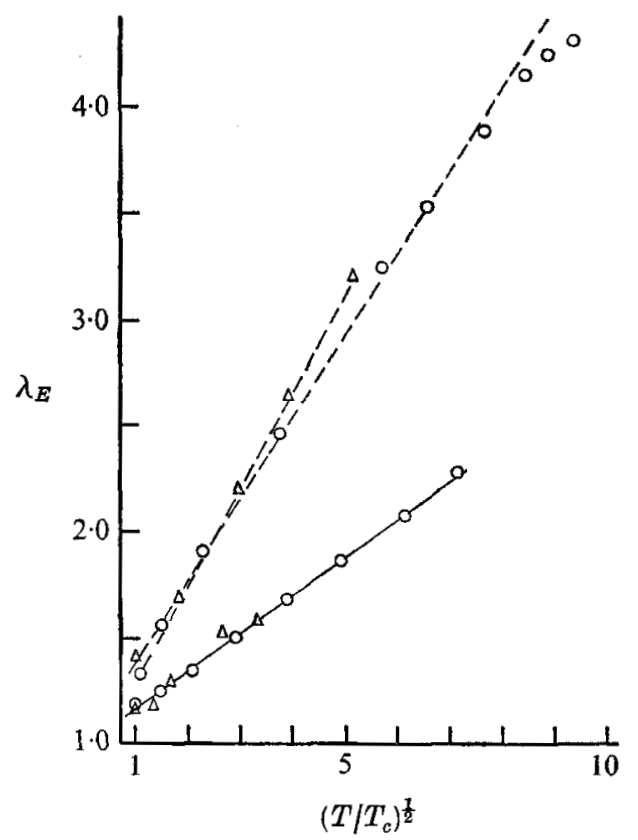

(b)

Figure 3. Variation of the end cell size with Taylor number. (a) Both end plates rotating. (b) Top surface free, bottom end plate rotating. $O$, fluid $1 ; 0$, fluid 2 ; $\triangle$, fluid 3 ; ,$- \eta=0.727 ;---, \eta=0.505$.

where $0 \cdot 13 \leqslant B \leqslant 0 \cdot 18$. The variations in the constants $A$ and $B$ are due to the different fluid viscosities. These variations are $\pm 8.9 \%$ in the mean slope for $\eta=0.505$ and $\pm 14.8 \%$ for $\eta=0.727$.

A non-rotating end boundary means that the end plate, which again covers almost the entire gap between both cylinders, is attached to the non-rotating outer cylinder. With this configuration a sink (fluid moving radially inwards) is 

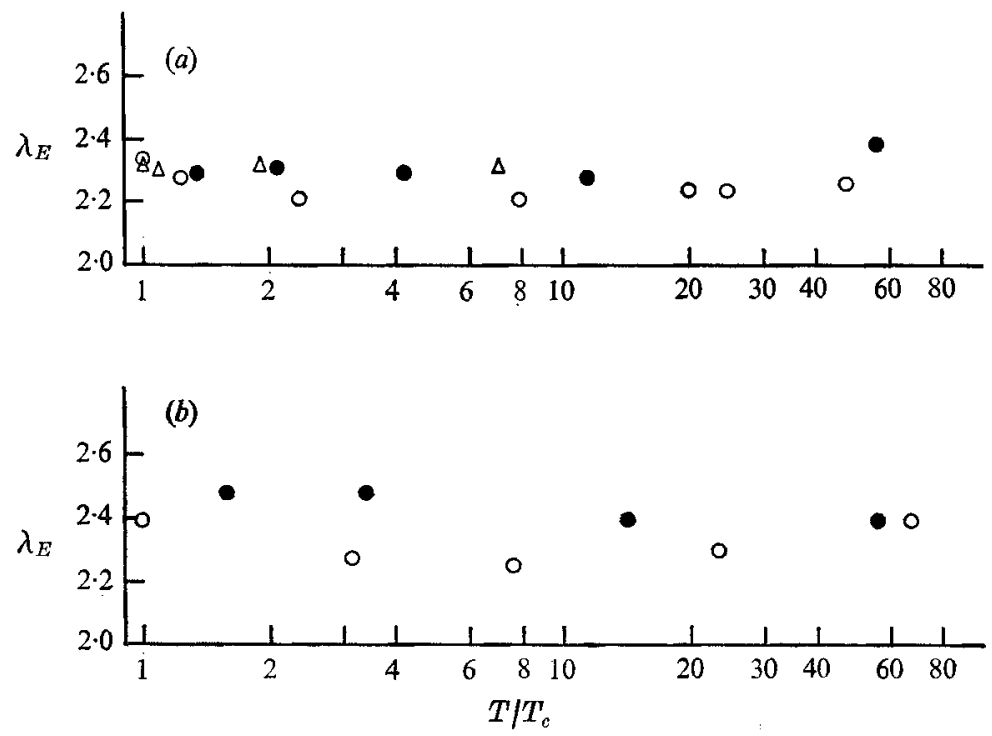

FigURE 5. Variation of end cell size with resting end plates. (a) Both end plates resting. (b) Upper surface free, bottom end plate resting. $\eta=0.727$. $O$, fluid 1 ; $O$, fluid 2; $\triangle$, fluid 3.

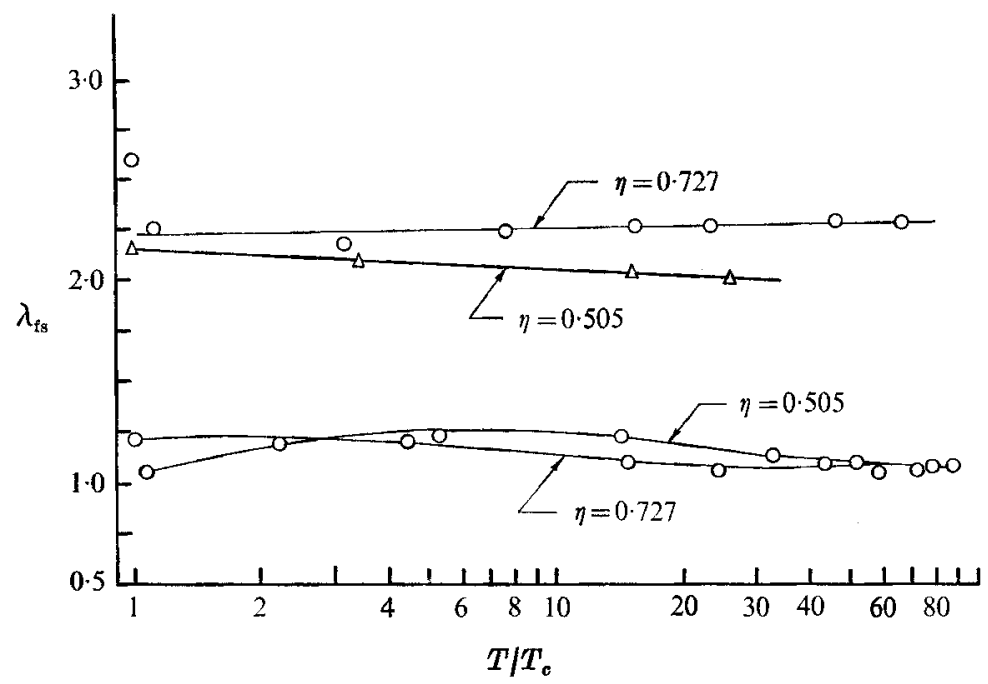

Figure 6. Variation of size of free end cells or rings with Taylor number. Upper curves for a ring, lower for a single cell. $O$, fluid $2 ; \Delta$, fluid 3 .

always formed adjacent to the end plate. Consequently a full ring appeared at each end of the fluid column. A typical example of the flow with a non-rotating end boundary is shown in figures $4(a)-(e)$ (plate 2). For photographs of the flow when neither boundary is rotating see Burkhalter (1972). In figure 4 it appears that the size of the end ring at the bottom remains unchanged over a large range of Taylor numbers. Measurements of the wavelength $\lambda_{E}$ are presented in figure 5 . Within the accuracy of the measurement, the size of the end ring does not depend 
on the Taylor number. However, the end ring is in general about $10 \%$ larger than the other rings in the fluid which are free from boundary effects.

A free surface boundary at the top of the fluid column produces either a sink or a source at the liquid-air interface. Qualitatively, a source is formed by fluids of lower viscosity at low rotation rates. A sink, always accompanied by a full ring, is formed by fluids with higher viscosities and higher rotation rates. Typical examples of free-surface cells have been shown in figures 2 and 4 . Both figures show the apparatus with an air gap of $1 \mathrm{~cm}$ between the fluid surface and the top end plates, which are of the same type as the bottom end plates. The top ring or cell under a free surface does not change with Taylor number, as in the case of non-rotating ends. In figure 6 , measured sizes of both full rings and single cells for both $\eta$ under a free surface are shown. Note that the size of the end rings or cells is slightly larger than that of the rings or cells in the rest of the fluid column. This was also the case when the end plates were non-rotating.

\section{Analysis of wavelength variations}

An analysis of the variations of the size of the end cells and consequent conclusions regarding the effective wavelength yield pertinent information concerning the variation of the wavelength in infinite cylinders with the same radius ratios as in these experiments. All experimental evidence obtained in finite cylinders has to be compared with theoretical results which pertain to an infinite cylinder with a comparable $\eta$. We now make use of the definition of the wavelength for a finite fluid column and recall that this definition does not account for end effects and thereby approximates the infinite cylinder case. If we assume that the actual wavelength $\lambda$ can be approximated by $\lambda_{\mathrm{fi}}=L / N d$ and note that the number of cells did not vary in any of the experiments as long as the flow was singly periodic, then it follows that the wavelength $\lambda$ does not vary for Taylor numbers less than $80 T_{c}$. The range up to which singly periodic flow can be maintained depends on the gap width and the end boundaries. Singly periodic flow was observed up to $80 T_{c}$ with fluid 1 with $\eta=0.505$ as well as $\eta=0.727$ with a free upper surface. A determination of the range of singly periodic flow as a function of the gap width was not attempted since there is no clear-cut point of transition from singly to doubly periodic flow.

The uncertainty $\Delta \lambda / \lambda$ of the measurements is $\pm 2 \%$. This simply means that, over the Taylor number range under consideration, if the wavelength had increased or decreased by $2 \%$ or more, then the number of cells would have changed by one cell or more. The rate of change of the wavelength with Taylor number is $d \lambda / d\left(T / T_{c}\right)=0 \pm 0.00048$ up to $80 T_{c}$. This conclusion concerns singly periodic flow. Actual changes in the number of cells occurred at Taylor numbers greater than $4000 T_{c}$ as will be discussed later. The rate of change of the wavelength with Taylor number which follows from figure $8(j)$ (plate 4), where the flow is turbulent, is $d \lambda / d\left(T / T_{c}\right)=+1.2 \times 10^{-5} \pm 0.6 \times 10^{-5}$. The positive sign of the rate of change is significant; the wavelength increases, but in order to do so the flow has to be turbulent.

We can likewise find the wavelength in the infinite cylinder by using the 
effective wavelength as a measure for $\lambda$. The experiments with non-rotating end boundaries show that the size of the end cells does not vary and consequently $\lambda_{\text {eff }}=$ constant. This is fairly obvious from figures $4(a)-(e)$. These experiments show, furthermore, that the non-rotating end boundaries behave as the freesurface boundary does. Therefore, a finite fluid column with non-rotating end boundaries imitates a finite fluid column with two free surfaces at the ends. This would certainly be the best experimental approximation to the infinite cylinder case. From the observation that with non-rotating ends neither the end cell size $E$ nor the number of rings $N$ changed, we can therefore conclude that the wavelength in an infinite cylinder would not change either, for singly periodic flow up to $80 T_{c}$. Since $\Delta E=0$, it follows from (2) that the uncertainty of this result is again approximately $\pm 2 \%$ and that the rate of change of the wavelength with Taylor number is again $d \lambda / d\left(T / T_{c}\right)=0$ with an uncertainty of about $\pm 0 \cdot 0005$.

The experiments with rotating end boundaries make it quite obvious (see figures $2(a)-(e))$ that in this case the effective wavelength of the rings free from end effects decreases. For example in figure $2(e), \lambda_{\text {eff }}=1.88$. In other experiments with shorter fluid columns $\lambda_{\text {eff }}$ has been reduced to $1 \cdot 51$; for more detail see Burkhalter (1972). On the other hand, with constant fluid column length, the wavelength $\lambda_{\mathrm{f}}$ remained constant in all experiments with rotating end boundaries since the number of rings never changed. The question arises now as to whether the observed decrease in $\lambda_{\text {eff }}$ under these circumstances is due to a true decrease of the wavelength $\lambda$ that might occur in an infinite cylinder, or whether this is caused by an effective reduction of the fluid column length caused by the growth of the end cells during the experiments.

Information concerning this problem can be obtained from experiments with fluid columns of different lengths. Suppose that a ring free from end effects would naturally shrink with increased Taylor number by the amount $\Delta$. This must necessarily cause an equivalent growth of the bottom end cell, since the entire column has to be filled with vortices. Provided that there is little interaction between the size of the free rings and the size of the end cells, the growth of the end cell would then be proportional to the number $n$ of rings on top of it, producing a variation of the end cell size of $n \Delta$. Since the number of rings is basically determined by the length of the fluid column, the end cells of long columns at a given supercritical Taylor number should therefore be longer than those of short fluid columns at the same supercritical Taylor number. Figure $7(a)$ shows the end cell size $\lambda_{E}$ for fluid columns 20,40 and $86 \cdot 30 \mathrm{~cm}$ long. All fluid columns had free upper surfaces since this was the easiest way to vary the column length. As can be seen in figure $7(a)$, the end cell size varies very little with column length; the increase of $\lambda_{E}$ for $L=86.30 \mathrm{~cm}$ is certainly not four times the increase of $\lambda_{E}$ for $L=20.0 \mathrm{~cm}$. We also note on figure $7(a)$ that the variation of $\lambda_{\text {eff }}$ with Taylor number in the case of the long column is much smaller than with the shortest column. As can be seen, the slope of the $\lambda_{\text {eff }}$ versus Taylor number curve will approach zero if the infinite cylinder case is approached. This means that the wavelength $\lambda_{1}$ actually does not vary and that the observed decrease of $\lambda_{\text {eff }}$ is caused by increases in the end cell size. We arrive at the same conclusions if we 


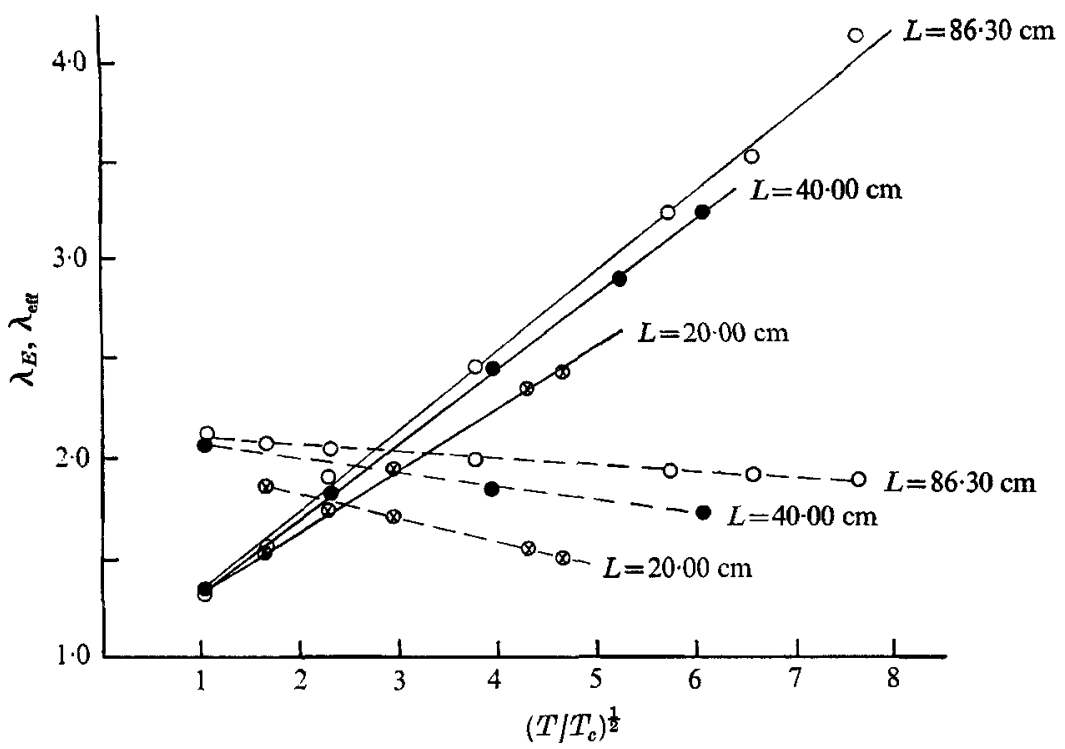

(a)

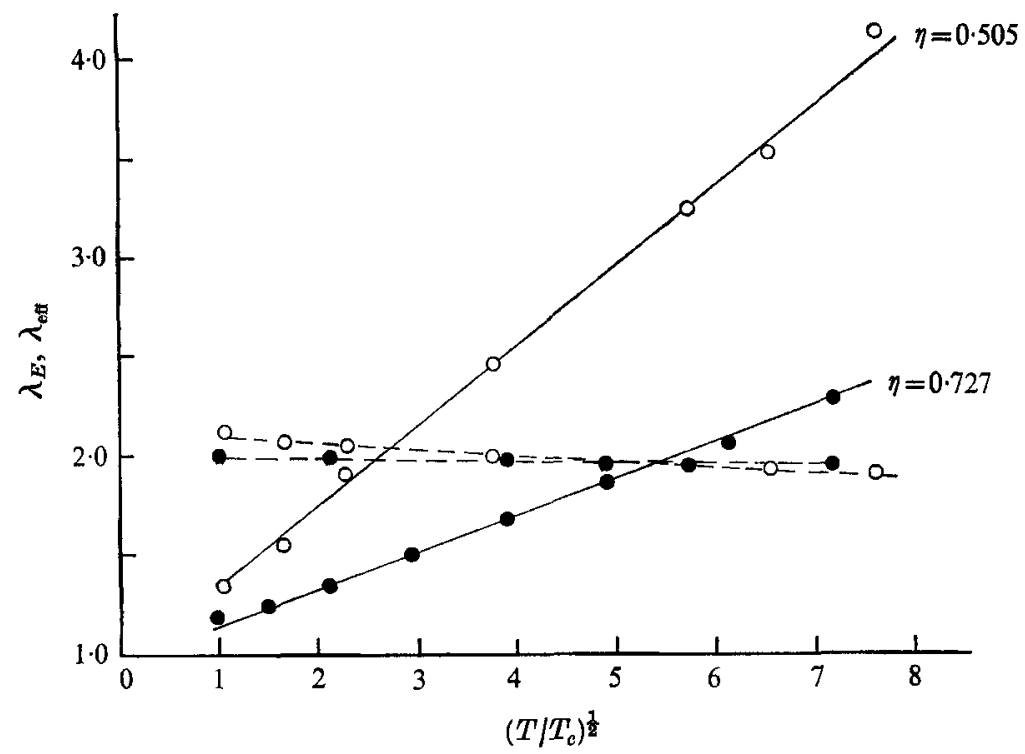

(b)

FIGURE 7. Variation of end cell size and $\lambda_{\text {eff }}$ for fluid 2. (a) For different fluid column lengths; $\eta=0-505$. (b) For different radius ratios. - - , $\lambda_{E} ;---, \lambda_{\mathrm{er}} \cdot$

study the variations of the end cell size and the effective wavelength as a function of radius ratio $\eta$, see figure $7(b)$. Large $\eta$ means many rings if the fluid column length is maintained, as is the case here. Many rings should, if the rings actually shrink, cause larger end cells as was discussed before. However, $\lambda_{E}$ for $\eta=0.727$ is much smaller than $\lambda_{E}$ for $\eta=0 \cdot 505$. Also, as in the case with different column lengths, the rate of change of the effective wavelength with Taylor number 
approaches zero with increasing $\eta$, which means that as $\eta$ increases, the infinite cylinder case is more closely approximated. The inference is again that the wavelength in an infinite cylinder would not change with Taylor number.

\section{Wavelength variation at very high supercritical Taylor numbers}

The previous discussion concerning the wavelength variations with Taylor number was applicable to singly periodic flow, where no waves in the azimuthal direction are present. We now ask the question: what changes (if any) occur in the effective wavelength, end cell growth, etc., at very high supercritical Taylor numbers where the flow is doubly periodic or turbulent?

To answer this question it was necessary to resort to fluid 1, of lower viscosity, in order to generate very high supercritical Taylor numbers. Representative photographic results of one of the experiments in the very high supercritical Taylor number regime are shown in figure 8 (plates 3 and 4). At low Taylor numbers $\left(T / T_{c}<80\right)$ fluid 1 behaved in exactly the same manner as the other two fluids used in the experiments for the case with rotating end plates (see figures $8(a)-(d))$. The end cells increased in size, as before, thereby causing a decrease in the effective wavelength. As the Taylor number was increased in a quasi-steady fashion past $T / T_{c}=80$, some interesting phenomena were observed. Although not discernible in the photograph, figure $8(d)$, there developed very slight oscillations or disturbances in the rings primarily associated with the sources. As the Taylor number was quasi-steadily increased to $T / T_{c}=124 \cdot 8$, figure $8(e)$, the disturbances in the flow became more apparent and visible. Note in figure $8(e)$ that the sources are tilted very slightly or have very small disturbances superimposed on them while the sinks (the darker lines) still remained laminar, straight and perfectly parallel to the horizontal. In addition, the end cells have continued to grow, causing a further decrease in the effective wavelength. Note that at about the Taylor number where the disturbances firstappeared in thesources, figure $8(d)$ or $(e)$, there are small perturbations in the end cells (see the bottom end cell, figure $8(e)$ ). Apparently the disturbances in the end cells are created by the end boundaries; however, these disturbances do not necessarily cause the disturbances to the sources since they both appear at about the same Taylor number. As the Taylor number was quasi-steadily increased still further to $T / T_{c}=186 \cdot 2$, figure $8(f)$, the disturbances on the sources became clearly visible as well as those in the end cells. It is remarkable, however, that the sinks are still laminar, straight, thin and perfectly parallel to the horizontal even though the sources are wavy and somewhat erratic. At a Taylor number of $T / T_{c}=269 \cdot 5$, all the rings are wavy, as shown in figure $8(g)$. The sinks have also become slightly wavy and the end cells are filled with disturbances created by the end plates. It was at this point, figure $8(g)$, that the flow is completely doubly periodic. That is, finite tangential waves are now superimposed upon all the rings. Another interesting feature noted in figure $8(g)$ is that the end cell has ceased to grow. As doubly periodic flow is established in the fluid column, the growth of the end cell stops. If the Taylor number is increased still further as in figure $8(h)$, the frequency of the tangential waves becomes higher, the sinks 
thicken and the end cells actually decrease in size. Note in figure $8(h)$ that the sources, though very wavy, are still discernible and discrete. In figure $8(i)$, where $T / T_{c}=2305$, the source lines have disa ppeared and the flow can only be described as being turbulent. Individual tangential waves are no longer discernible and the end cells have continued to shrink.

Up to this point, figure $8(i)$, the number of rings or cells in the fluid column remained constant. As the Taylor number was increased to $T / T_{c}=4695$, figure $8(j)$, the number of rings decreased to 23 , thereby increasing the effective wavelength as well as $\lambda$. The two rings that suddenly disappeared were the ones adjacent to the end cells. For the wavelength to increase it is apparently a necessary condition that the flow be first doubly periodic. As the Taylor number was increased to $T / T_{c}=7175$ and finally to 11330 , figures $8(k)$ and $(l)$, more rings disappeared (again adjacent to the end cells), until finally, figure $8(l)$, only 18 full rings were left (not counting end cells). Note that the size of the end cells has increased again. It is remarkable that at this extremely large Taylor number, where the flow is highly turbulent, the Taylor vortices are still clearly visible in the flow. Qualitatively the same observation was made by Schultz-Grunow \& Hein (1956), who also observed the decrease in the number of rings, as did Pai (1943). The maximum rotation rate was reached when figure $8(l)$ was taken and it is not known if more rings would have disappeared at higher Taylor numbers. The effective wavelength in figure $8(l)$ is $\lambda_{\text {eff }}=2 \cdot 54$. Repeating such an experiment exactly in a quasi-steady way does not necessarily produce the same wavelength at the same highly supercritical Taylor number. However, there is a definite decrease in the number of rings at large supercritical Taylor numbers which makes it necessary that the average wavelength be definitely larger than the critical wavelength. Much more information concerning the wavelength at large supercritical Taylor numbers will be presented with the results of suddenstart experiments, see Burkhalter \& Koschmieder (1973). The indeterminacy of the measured wavelengths makes the results of the experiments at highly supercritical Taylor numbers quite different from the results with laminar, singly periodic flow. In the singly periodic case the size of the rings as well as the size of the end rings were strictly reproducible, as long as the experiment was performed in a quasi-steady way.

\section{Conclusions}

The experiments described above deal with a comparatively simple problem, namely the question of what happens to the size of the Taylor vortices if the Taylor number is increased in a quasi-steady way beyond the critical Taylor number. We have simplified the problem further by restricting the investigation to singly periodic flow. There seems to be no theoretical investigation available which would predict how the wavelength of Taylor vortices should vary under these conditions. Coles (1965) has shown experimentally that the relations between the wavelength, number of tangential waves and Taylor number are very complex in the case of doubly periodic flow. The results of the above experiments show that the wavelength of the Taylor vortices which one would observe 
in an infinitely long cylinder does not vary with the Taylor number as long as the flow is singly periodic, in these experiments up to $80 T_{c}$. With regard to the effective wavelength it was found that $\lambda_{\text {eff }}$ does not vary if either both end plates are non-rotating or the upper end is free and the lower one non-rotating. The case of two non-rotating end plates seems to approximate the hypothetical case with two free ends and is therefore the best experimental approximation to the infinitely long cylinder. It was, on the other hand, quite obvious that the effective wavelength decreases substantially with increased $T$ if either both end plates rotate or the upper one is free and the lower one is rotating. Control experiments made with different fluid column lengths indicate, however, that in the case with rotating boundaries the wavelength in an infinite cylinder would not vary with Taylor number either. This can only be inference; it needs a theoretical analysis of the variation of the size of the end cells with Taylor number to make these observations conclusive.

This work was supported in part by NSF Grant GA-11484. The work of J.E. Burkhalter was done in partial fulfillment of the requirements of a $\mathrm{Ph} . \mathrm{D}$. degree.

\section{REFERENCES}

Burkhalter, J. E. 1972 Ph.D. dissertation, University of Texas, Austin. Burkhatter, J. E. \& Koschmieder, E. L. 1973 Submitted for publication. Coles, D. 1965 J. Fluid Mech. 21, 385.

Donneliy, R. J. \& Fultz, D. 1960 Proc. Roy. Soc. A 258, 101.

Donneliy, R. J. \& Schwarz, K. W. 1965 Proc. Roy. Soc. A 283, 531.

Kogelman, S. \& DiPrima, R. C. 1970 Phys. Fluids 13, 1.

Koschmreder, E. L. 1973 Adv. Chem. Phys., to appear.

PaI, S. I, 1943 N.A.C.A. Tech. Note, no. 892.

Roberts, P. H. 1965 Proc. Roy. Soc. A 283, 550.

Schulaz-Grunow, F. \& Hern, H. 1956 Z. Flugwiss. 4, 28.

SNYDER, H. A. 1969 J. Fluid Mech. 35, 273.

StUART, J. T. 1971 Ann. Rev. Fluid Mech. 3, 347. 